\title{
Overcoming Operational Differences to Attain a National Picture for Novel Threats
}

\author{
Michael Coletta*, Achintya N. Dey, Matthew Miller, Peter Hicks and Umed Ajani \\ Centers for Disease Control and Prevention, Atlanta, GA, USA
}

\section{Objective}

Demonstrate that information from disparate syndromic surveillance (SyS) systems can be acquired and combined to contribute to national-level situational awareness of emergent threats.

\section{Introduction}

The May arrival of two cases of Middle East Respiratory Syndrome (MERS) in the US offered CDC's BioSense SyS Program an opportunity to give CDC's Emergency Operations Center (EOC) and state-and-local jurisdictions an enhanced national picture of MERS surveillance. BioSense jurisdictions can directly query raw data stored in what is known as "the locker." However, CDC cannot access these data and critical functions, like creating ad-hoc syndrome definitions within the application are currently not possible. These were obstacles to providing the EOC with MERS information. BioSense staff developed a plan to 1) rapidly generate query definitions regardless of the locally preferred SyS tool and, 2) generate aggregate reports to support the national MERS response.

\section{Methods}

In consultation with state and local SyS epidemiologists, a common-use set of five MERS-specific query definitions were developed. The definitions focused on:

sensitivity,

specificity,

travel,

symptoms, and

ICD diagnosis codes.

Jurisdictions had various operating environments so all definitions were operationalized in SQL, R-Script, SAS, and ESSENCE query languages. The definitions and deployment guidance were provided to state and local jurisdictions. Also, a webinar was conducted to call for participation in MERS-like surveillance activities. CDC answered participants' questions and setup weekly data reporting, which was collated into reports for the EOC.

\section{Results}

Call for Participation in MERS-like Surveillance Webinar: 192 people attended, representing $60 \%$ of registrants. $35 \%$ of webinar participants completed an exit survey. $63 \%$ of exit survey respondents reported the activity directly applicable to their work. $78 \%$ felt they had a better awareness and understanding following the webinar. $42 \%$ of exit survey participants reported an anticipated change in behavior, while $68 \%$ reported the webinar contained useable ideas and techniques.

Participation and Data Sharing Among CDC and Jurisdictions: Of the 11 jurisdictions providing data-sharing feedback, $64 \%$ were interested in sharing data and $9 \%$ were not.

In the end, 15 jurisdictions-6 large cities and 9 states-participated in MERS-like surveillance. They represented 822 facilities nationwide. Ten of 15 jurisdictions used a local SyS system to gather and report data. Surveillance activities began May 12 and continued through July 31. Participating jurisdictions shared data only with CDC; they did not share with each other.

\section{Conclusions}

Despite limitations and imperfections, surveillance reports of MERS-like visits enhanced the MERS national surveillance picture. CDC and partner jurisdictions quickly developed a novel, standardized set of definitions that were widely shared. Though limited by the use of differing systems, the community succeeded in providing CDC with MERS-like surveillance data. This national activity will inform future development of SyS applications.

Several operational limitations persisted and should be discussed among the community. The 15 participating jurisdictions covered a substantial population but did not approach full or uniform national coverage. Despite participants' fairly quick response, activating the surveillance method was too slow for the MERS response. Thus, SyS data became ancillary to EOC operations. Participants never agreed how to share MERS-like surveillance data beyond CDC; no data were shared among jurisdictions. However, many stakeholders viewed this attempt to enhance the national MERS surveillance picture as a model to expand, and a success that may improve trust among jurisdictions giving hope for providing meaningful national SyS data in the future.

\section{Keywords}

Syndromic Surveillance; MERS; BioSense

Acknowledgments

CDC DHIS, CDC EOC, Participatory and Consultant State and Local Jurisdictions

\section{* Michael Coletta}

E-mail: mac0@cdc.gov 